SEVERINO DENICIO GONÇALVES DE SOUSA

\title{
EFEITO DO HIPOTIROIDISMO E DA OOFORECTOMIA NA FUNÇÃO CARDÍACA DE RATAS
}

Dissertação apresentada ao Programa de Pós-Graduação em Ciências Morfofuncionais do Departamento de Anatomia do Instituto de Ciências Biomédicas da Universidade de São Paulo para obtenção do Título de Mestre em Ciências.

Área de concentração: Ciências Morfofuncionais

Orientadora: Drª. Maria Luiza Morais Barreto de Chaves

Versão corrigida. A versão original eletrônica, encontra-se disponível tanto na Biblioteca do ICB quanto na Biblioteca Digital de Teses e Dissertações da USP (BDTD);

São Paulo

2017 


\section{RESUMO}

\section{SOUSA, S. D. G. Efeito do hipotiroidismo e da ooforectomia na função}

cardíaca de ratas. 84 f. Dissertação (Mestrado em Ciências Morfofuncionais) Instituto de Ciências Biomédicas, Universidade de São Paulo, São Paulo, 2017.

Este trabalho avaliou, em corações isolados de ratas Wistar, através do sistema de perfusão ex vivo - Langendorff, diferentes parâmetros de função cardíaca, frente a um evento de Isquemia e reperfusão. Foram estabelecidas duas situações experimentais: a primeira avaliou o efeito do hipotireoidismo na condição clínica (Hipo) e no hipotireoidismo subclínico (HTS) sobre os parâmetros de função cardíaca. O segundo avaliou o efeito da ooforectomia sobre os mesmos parâmetros (OO). Após a realização dos protocolos experimentais, os animais foram decapitados, o coração rapidamente retirado e perfundido com solução de Krebs-Henseleit durante 30 min de estabilização. Após esse período os parâmetros de Pressão Desenvolvida pelo Ventrículo Esquerdo (LVDP),Pressão Diastólica Final (EDP) e Pressão de Perfusão foram similares entre os grupos. Porém, a Primeira Derivada Positiva das Pressões $(+d P / d t)$, Primeira Derivada Negativa das Pressões $(-d P / d t)$ e frequência cardíaca foram menores no grupo Hipo, em relação ao controle. Após o período de estabilização, os corações foram submetidos a um período de 20 min de isquemia (fluxo zero), com posterior restauração do fluxo (período de reperfusão). Após a reperfusão, os corações Hipo apresentaram EDP, +dP/dt e -dP/dt mais próximos aos encontrados no respectivo período de estabilização, quando comparados as demais grupos. Os grupos HTS ou OO não apresentaram diferenças significativas quanto a esses parâmetros, indicando que essas duas condições foram insuficientes para alterar a recuperação cardíaca após um evento isquêmico.

Palavras-chave: coração isolado, isquemia e reperfusão, hipotireoidismo, hipotireoidismo subclínico e ooforectomia. 


\begin{abstract}
SOUSA, S. D. G. 2017.Effect of hypothyroidism and oophorectomy in the cardiac function of female rats. 84 p. Master thesis (Morphological Sciences) - Instituto de Ciências Biomédicas, Universidade de São Paulo, São Paulo, 2017.
\end{abstract}

This work evaluated, in isolated hearts of Wistar rats, through the perfusion system ex vivo - Langendorff, different parameters of cardiac function, after Ischemia-reperfusion (I-R). Two experimental situations were established: the first evaluated the effect of hypothyroidism on the clinical condition (Hypo) and subclinical hypothyroidism (HTS) on the parameters of cardiac function. The second evaluated the effect of oophorectomy on the same parameters (OO). After performing the experimental protocols, the animals were decapitated, the heart rapidly withdrawn and perfused with Krebs-Henseleit solution for 30 min of stabilization. After this period the parameters of Left Ventricle Developed Pressure (LVDP), Final Diastolic Pressure (EDP) and Perfusion Pressure were similar between groups. However, the First Positive Pressure Derivative (+ dP / $\mathrm{dt}$ ), First Negative Derivative Pressures (-dP / dt) and heart rate were lower in the Hipo group, in relation to the control group. After the stabilization period, the hearts were submitted to a 20 min period of ischemia (zero flow), with subsequent flow restoration (reperfusion period). After 35 min reperfusion, Hipo hearts presented EDP, $+\mathrm{dP} / \mathrm{dt}$ and $-\mathrm{dP} / \mathrm{dt}$ closer to those found in the respective stabilization period, when compared to the other groups. The HTS or OO groups did not present significant differences in these parameters, indicating that these two conditions were insufficient to alter the cardiac recovery after an ischemic event.

Key words: isolated heart, ischemia and reperfusion, hypothyroidism, subclinical hypothyroidism and oophorectomy. 


\section{INTRODUÇÃO}

O coração corresponde a uma bomba contrátil propulsora que, frente a um sistema fechado formado pela rede vascular, gera uma pressão sanguínea, a qual é necessária para o estabelecimento de um fluxo sanguíneo, e com ele, a adequada perfusão de tecidos e órgãos. $O$ bombeamento efetivo de sangue depende de um conjunto de forças, entre elas a força contrátil exercida a cada ciclo cardíaco pela musculatura ventricular, a qual no nível celular é absolutamente dependente das concentrações intracelulares do íon cálcio. Muitos hormônios atuam sobre o coração, modulando o seu trofismo, o seu metabolismo e a sua função contrátil. Assim, alterações nas concentrações plasmáticas de glicocorticóides, noradrenalina, angiotensina II, insulina, hormônios sexuais masculinos e femininos, além dos hormônios tiroideanos atuam diretamente no tecido cardíaco, podendo trazer repercussões funcionais marcantes, tanto em situações normais como após processos de injúria resultantes de Isquemia (I), acompanhados ou não por Reperfusão (R).

\section{1- Isquemia/Reperfusão (I/R)}

Doenças cardiovasculares, entre elas aquelas que culminam com a deficiência de irrigação do tecido levando ao infarto do miocárdio, correspondem ainda nos dias de hoje a uma das principais, se não a principal, causa de óbitos na população em geral.

A isquemia miocárdica é resultante de um comprometimento do fluxo sanguíneo coronariano, gerado a partir de um desequilíbrio entre a oferta e a demanda de oxigênio. No entanto, se a isquemia decorrente da falta de irrigação pode levar ao infarto, a injúria tecidual que se desenvolve como consequência a episódios de isquemia-reperfusão (I/R), nos quais ocorre a restauração do fluxo sanguíneo aos tecidos ou órgãos isquêmicos, pode levar a efeitos até mais deletérios do que aqueles decorrentes da própria isquemia. Assim, embora a ausência de fluxo sanguíneo contribua para a fisiopatologia de diversas doenças, como o infarto do miocárdio e a insuficiência vascular periférica, a restauração do fluxo sanguíneo ou a reperfusão, mesmo que 
necessária, pode por si só agravar o dano celular isquêmico (SILVA JR et al., 2002).

Entre vários fatores que contribuem para essa injúria celular encontra-se o fato do restabelecimento do fluxo sanguíneo após um evento isquêmico ocorrer de modo não-uniforme para todo o tecido, resultando na restauração "caótica" do fluxo tissular, o que pode levar a um círculo vicioso de disfunção endotelial vascular, com redução da perfusão local, edema, entre outras complicações (EVORA et al., 1996). Em teoria o processo é muito simples, a falta de oxigenação e de substratos metabólicos adequados diminui rapidamente a energia disponível para a célula, levando à lesão celular, que pode ser de natureza reversível ou irreversível. Na prática, o processo é muito complexo, uma vez que a extensão da lesão é determinada por vários fatores, como: gravidade da isquemia (baixo fluxo vs fluxo zero), duração da isquemia, sequência temporal da isquemia (isquemia curta seguida de isquemia longa), mudanças no ambiente físico e metabólico (normotermia vs hipotermia; conteúdo de glicogênio no miocárdio antes da isquemia, composição do perfusato), bem como a resposta inflamatória (PANTOS, 2006).

Ao longo dos últimos anos, os estudos sobre fenótipos de maior tolerância contra a isquemia e reperfusão tornou-se uma importante ferramenta na busca de evidências sobre a resposta adaptativa do coração a um estresse isquêmico. Vários paradigmas de cardioproteção foram identificados e são extensivamente estudados na esperança de encontrar novos alvos terapêuticos para o miocárdio isquemiado. Sabe-se que alguns hormônios desempenham um papel importante na resposta do coração à isquemia. Neste contexto, as alterações hormonais têm sido investigadas na tolerância do miocárdio ao estresse isquêmico (PANTOS et al., 2004).

\subsection{Hormônios Tiroideanos}

Doenças da glândula tireoide, associadas a alterações das concentrações plasmáticas de hormônios tiroideanos, estão entre as mais frequentes doenças endócrinas encontradas no mundo. Estimativas indicam que essas doenças atingem entre 9\% e 15\% das mulheres adultas, e em menor porcentagem os homens (CANARIS et al., 2000). Ainda, dados de 2010 indicam que aproximadamente $15 \%$ da população brasileira é acometida por 
doenças que afetam a glândula tireoide (IBGE, 2010), sendo que alterações das concentrações desses hormônios são capazes de aumentar o fator de risco para o desenvolvimento e progressão das doenças cardiovasculares (SCHMIDT-OTT; ASCHEIM, 2006). Desta forma, fica evidente a importância de se investigar com maior profundidade a ação desses hormônios sobre 0 coração.

A tireóide é uma glândula localizada na região cervical, anteriormente à traquéia, acima da cartilagem cricóide e inferiormente à cartilagem tiroidea. Esta glândula, que se apresenta dividida em dois lobos, direito e esquerdo, unidos por um istmo, é constituída principalmente por dois tipos de células com origem embriológica distinta: as células $\mathrm{C}$ ou parafoliculares - produtoras de calcitonina, que compreendem pouco mais de $1 \%$ das células da tireoide, e as células foliculares - produtoras de tiroxina (T4), que se apresentam em maior número. As células folicularesse organizam de forma circular, o folículo, o qual envolve uma matriz gelatinosa denominada de coloide. É no coloide o local onde ocorre a ligação do iodeto aos aminoácidos de tirosina, processo denominado de iodinação (incorporação de iodo). Conforme o conteúdo e distribuição de iodeto, uma série de iodotironinas, a partir dos aminoácidos de tirosina, podem ser formadas, das quais a tiroxina (T4) possui quatro iodos ligados ao seu anel benzênico e a triiodotironina (T3) com somente três iodos.

A síntese e secreção dos hormônios tireoidianos (HTs) é regulada pelo eixo hipotálamo-hipófise-tireóide.Em resumo, o hipotálamo sintetiza e secreta o hormônio liberador da tireotrofina (TRH), que alcança a região anterior da hipófise (adenohipófise) e estimula a síntese do hormônio tireotrófico (TSH), que se liga aos receptores de TSH (TSH-R) presentes nas células foliculares da tireoide, ativando a síntese e secreção dos HTs.

Cerca de $80 \%$ dos hormônios tireoideanos secretados pela glândula tireóide apresentam-se como T4 e os $20 \%$ restantes como T3, o qual é considerado 0 hormônio tireoidiano biologicamente ativo (SAPIN; SCHLIENGER 2003). O T4 é convertido a T3 por ação de enzimas denominadas deiodinases ou desiodases, localizadas em diversos tecidos do organismo. Assim, embora o T4 esteja presente em maior quantidade, é o T3 o responsável pela maioria das atividades biológicas, agindo em praticamente todas as células do organismo, nas quais modula diretamente a expressão de 
diferentes genes e proteínas com funções estruturais, funcionais, metabólicas. Embora hoje sejam já bem reconhecidas ações não-genômicas dos HTs, rapidamente ativadas, as ações genômicas clássicas são mediadas pela ligação a receptores nucleares (TRs), sendo o T3 a principal isoforma responsável por estes efeitos, uma vez que este apresenta uma afinidade de ligação maior quando comparada à do T4 (BEN SANDLER et al., 2004).

\subsection{Hormônios Tiroideanos e função cardíaca}

O tecido cardíaco corresponde a um dos principais alvos dos HTs, sendo muito sensível a alterações séricas ou locais dos mesmos (KENESSEY; OJAMAA, 2006; TAVARES et al., 2013). Os mecanismos celulares pelos quais os hormônios tireoidianos atuam sobre a função cardíaca sistólica e diastólica são complexos e em diferentes níveis. Os hormônios tireoidianos modulam a expressão e a função de várias enzimas e proteínas envolvidas no desempenho cardíaco, como o retículo sarcoplasmático $\mathrm{Ca}^{+2}$ ATPase (SERCA II), $\mathrm{Na}^{+} / \mathrm{K}^{+}$ATPase e as cadeias pesadas de alfa/beta-miosina. Assim, o T3 estimula a expressão do gene que codifica a cadeia pesada de miosina do tipo alfa (MHC- $\alpha$ ) e reprime o gene da $\beta-\mathrm{MHC}$, além de aumentar a expressão de outras proteínas contráteis que formam os filamentos finos, como a actina e a troponina I (DIECKMAN; SOLARO, 1990; SHAHRIVAR et al., 2016).

O efeito dos HT sobre a função cardíaca já tem sido intensamente explorado pela literatura. Entretanto, o papel destes hormônios em condições patológicas como aquelas que envolvem fenômenos de isquemia, infarto e insuficiência cardíaca ainda permanece a ser elucidado. Neste sentido, trabalhos realizados tanto em modelos experimentais com animais como em trabalhos clínicos, demonstram que os níveis séricos de T3 caem significativamente após uma injúria cardíaca, estando estes níveis associados a disfunção ventricular esquerda e a alteração em vários genes responsivos ao HT (OJAMAA et al., 2000; PANTOS et al., 2012). Assim, em humanos, uma queda significativa já é observada 48h após a ocorrência de infarto agudo do miocárdio (IAM) (EBER et al., 1995), como também após 6-24h a realização de cirurgia de revascularização miocárdica (HOLLAND et al.,1991).

Segundo Friberg et al. (2002), a diminuição dos níveis séricos de T3 pode então corresponder a um importante fator preditivo para mortalidade após um 
IAM, sendo que o tratamento com administração de T3 por quatro semanas, por outro lado, já se mostrou eficiente na melhora da função cardíaca e na normalização da maioria das alterações da expressão de genes-alvos cardíacos dos HT (OJAMAA et al., 2000). Ainda neste contexto, em estudo realizado com grupos de pacientes que sofreram IAM, com ou sem angina pectoris prévia, os autores relatam que a angina parece promover a diminuição dos níveis de HT, representando uma vantagem uma vez que, provavelmente ocorre redução na demanda de oxigênio miocárdico e diminuição da taxa metabólica. No entanto, os mesmos autores discutem que a emergência da falência cardíaca parece ocorrer com maior frequência em pacientes hipotireoideos, quando comparados a pacientes eutireoideos (FRIBERG et al., 2002).

A razão para a diminuição dos níveis séricos dos HT ainda não é bem estabelecida. No entanto, um dos mecanismos que é diretamente influenciado pela queda dos HT em períodos pós-isquêmicos é aquele relacionado ao manejo de cálcio do cardiomiócito, o qual pode vir a contribuir para um quadro denominado de myocardial stunning, uma redução reversível da função contrátil do coração após a reperfusão pós-isquêmica, devido a uma sobrecarga de cálcio (KRAUSE et al., 1989).

Davis e Davis (1993) descreveram que os hormônios tireoidianos podem alterar a função ventricular por agir sobre a atividade da $\mathrm{Ca}^{2+}$-ATPase do retículo sarcoplasmático. O ATP é responsável pela remoção do cálcio do citosol durante a diástole, permitindo, dessa forma, o desacoplamento do complexo actina-miosina, sendo de extrema importância para a função cardíaca diastólica (DILLMAN, 1990).

Já é bem conhecido na literatura que o excesso de HTs é capaz de elevar o débito cardíaco de 50 a 300\%, aumentando a frequência cardíaca de repouso, contratilidade e fração de ejeção. Por outro lado, o hipotireoidismo apresenta manifestações cardíacas clínicas opostas às do hipertireoidismo, como bradicardia, diminuição da contratilidade cardíaca e diminuição do débito cardíaco (KLEIN; DANZI, 2007;CROWLEY et al., 1977).

Dados do nosso laboratório, utilizando modelo de perfusão de coração isolado de ratos Wistar adultos, revelaram que a recuperação funcional pós-isquêmica foi melhor em animais tratados previamente com T3, quando comparados aos 
eutiroideos (TAVARES et al., 2013).Os animais tratados com T3 apresentaram um aumento da expressão do receptor AT2 e uma maior atividade da proteína quinase ativada por AMPK, os quais foram prevenidos após administração de PD123319, um antagonista do receptor AT2, havendo piora da função cardíaca. Embora os efeitos do hipertiroidismo no modelo de I-R já tenham sido previamente descritos, pouco se conhece em relação aos efeitos da deficiência de HTs sobre a função cardíaca, e é neste contexto que o nosso estudo se propõe a contribuir com o avanço do conhecimento nessa área.

O hipotireoidismo, como citado inicialmente, é uma condição relativamente comum, afetando em torno de $15 \%$ das mulheres no climatério e até $5 \%$ da população mundial (EMPSON et al., 2007). Em humanos, esta doença é classificada, de acordo com as manifestações clínicas, em hipotireoidismo propriamente dito, o qual pode se apresentar em diferentes intensidades, e hipotireoidismo subclínico, no qual as manifestações clínicas não são ainda aparentes, embora os níveis hormonais já se encontrem alterados.

O hipotireoidismo subclínico é definido como um aumento sérico dos níveis de hormônio estimulante da tiróide (TSH) acompanhado de níveis normais de T3 e T4 (Figura 1) (KARTHICK et al., 2013). Já o hipotireoidismo estabelecido, o qual tem sido bem mais estudado em relação ao primeiro, é definido por concentrações séricas de T3 e T4 abaixo dos valores normais, além dos valores de TSH também elevados.

Estimativas apontam que pelo menos $10 \%$ das mulheres após o período reprodutivo apresenta hipotireoidismo subclínico, (CANARIS et al., 2000; COOPER, 2008), mostrando ter este distúrbio uma importante relevância como fator de risco cardíaco na população em geral (MADATHIL et al., 2015). Embora esses dados sejam facilmente encontrados na literatura quando se analisa a população humana, não existem estudos em roedores mostrando a variabilidade das concentrações dos HT ao longo da vida, nem tampouco entre os gêneros. 


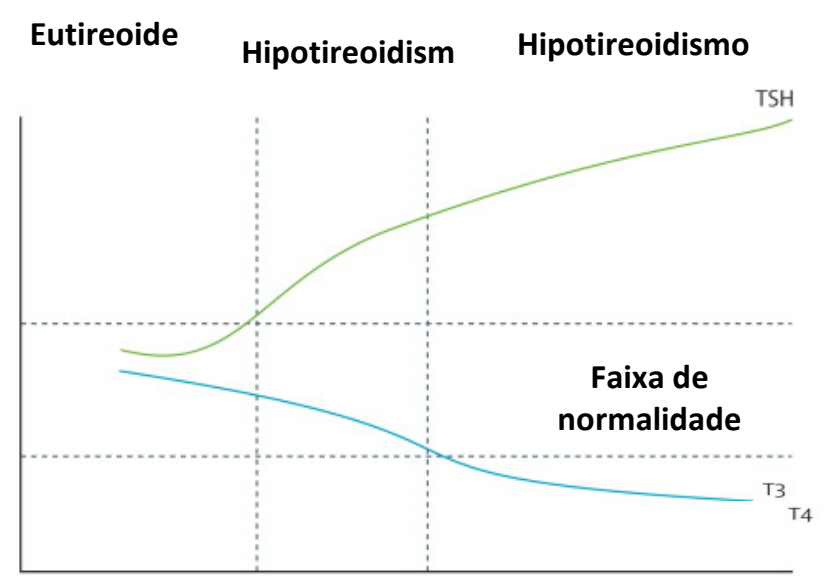

Figura 1. Gráfico representativo das distribuições hormonais nas diferentes condições de hipotireoidismo.

Fonte: Modificado de Yoshimi Ohga et al., 2002.

A disfunção diastólica, com maior tempo de relaxamento isovolumétrico, é a anormalidade cardíaca mais frequente em indivíduos com níveis de T3 e T4 normais e TSH sérico elevado (MONZANI et al., 2001; MEENA et al., 2012). Essas anormalidades geralmente decorrem, como já citado anteriormente, de mudanças na homeostase do cálcio, comprometendo a bioenergética cardíaca, que, no hipotireoidismo subclínico, é normalmente reversível com terapia com levotiroxina ou T4 (MADATHIL et al., 2015).

Embora os efeitos cardíacos associados às condições de hipotireoidismo subclínico ainda sejam incipientes, não se encontram dados na literatura mostrando como o coração, sob essas condições, responderia a situações de isquemia, ou seja, qual o grau de recuperação que o órgão apresentaria após eventos de isquemia, seguidos ou não de reperfusão. Isto se torna ainda mais intrigante se considerarmos a fase na qual o hipotireoidismo subclínico é mais prevalente, ou seja, após a diminuição nos níveis de hormônios sexuais femininos.

\subsection{Hormônios ovarianos}

Os ovários são órgãos pares em formato oval, localizados na cavidade pélvica e responsáveis pela produção dos gametas femininos. Além de seu papel na produção de gametas, os ovários também atuam como glândulas endócrinas produtoras e secretoras de hormônios esteroides, como os 
estrogênios, os andrógenos e os progestágenos, sendo cada um definido por sua atividade biológica e tendo a sua síntese controlada pela liberação pulsátil do hormônio de liberação de gonadotrofina $(\mathrm{GnRH})$. Quando o $\mathrm{GnRH}$ é liberado pelo hipotálamo, estimula a produção e secreção dos hormônios folículo estimulante (FSH) e luteinizante (LH) pela adeno-hipófise, e estes irão atuar estimulando as gônadas a produzirem e a secretarem os hormônios esteroides, que por sua vez agem sobre os seus respectivos receptores, isto é, receptores de estrogênio $(E R)$, receptores de progestina $(P R)$, e receptores de andrógenos (AR). O grupo dos estrogênios é formado por três potentes hormônios esteroides, sendo eles a: estrona, estriol e estradiol. Os estrogênios encontram-se em níveis elevados no sexo feminino desde o inicio ao final da vida reprodutiva (KNOWLTON; KORZICK, 2014). O estradiol é o mais potente entre os estrogênios e em geral circula em maiores concentrações. Por exemplo, os ovários de ratas secretam 5 a 8 vezes menos estrona do que estradiol ao longo do ciclo estral e da gravidez (SHAIKH, 1971; BLAUSTEIN, 2008; ASARIAN; GEARY, 2013).

Os hormônios estrogênicos agem através de uma série de vias de sinalização que possibilitam efeitos genômicos e não-genômicos. Os estrogênios transmitem a maioria das suas informações ao ligar-se aos seus fatores de transcrição, receptores intracelulares de estrogênio (ERa e ERß), sendo cada um codificado por um gene distinto (ESR1 e ESR2, respectivamente), apesar de apresentarem homologia quanto à sua sequência (HALL; COUSE; KORACH, 2001). Esses receptores são expressos em tecidos diferentes e são encontrados tanto no citosol como no núcleo. No entanto, também podem estar associados à membrana plasmática e à mitocôndria (HALL; COUSE; KORACH, 2001; LI et al., 2004). Fêmeas nocautes para esses receptores apresentam hipoplasia no trato reprodutivo, falta de desenvolvimento da glândula mamária e excesso de tecido adiposo, enquanto que machos apresentam degeneração testicular e disfunção epididimal (COUSE; KORACH; KORACH, 1999). No ano de 2005 um terceiro receptor de estrogênio, acoplado à proteína G (GPER ou GPR30) foi descrito e, assim como os demais clássicos receptores acoplados à proteína G, este apresenta 7 domínios transmembranares (REVANKAR, 2005). 
Além dos efeitos sobre as funções reprodutivas, os estrogênios desempenham papel significativo na regulação da homeostase esquelética, no metabolismo de lipídios e carboidratos, no equilíbrio de eletrólitos, além de atuarem sobre a função do sistema nervoso central e do sistema cardiovascular, citado em (NILSSON; GUSTAFSSON, 2011).

Neste sentido, a diminuição dos níveis de estrógeno ao final da vida reprodutiva, aprece estar associada ao aumento da incidência das doenças cardiovasculares, podendo aumentar de duas a três vezes a incidência de doenças cardíacas coronárias, incluindo o infarto do miocárdio (KANNEL et al., 1976; ROGER et al., 2011; KORZICK; LANCASTER, 2013).

\subsection{Hormônios ovarianos e função cardíaca}

Em mamíferos, os efeitos dos hormônios ovarianos estão presentes em todos os níveis da fisiologia cardiovascular, desde a duração do potencial de ação, à energia mitocondrial até à função contrátil do miócito cardíaco e relaxamento do coração como um todo (BLENCK et al., 2016; PARKS; HOWLETT, 2013). Tanto os cardiomiócitos como os fibroblastos cardíacos possuem receptores funcionais para grande parte dos hormônios esteroides sexuais, permitindo que estes hormônios regulem tanto a força contrátil da célula muscular, como a produção de colágeno, em processos de remodelamento (GROHÉ et al., 1997; PARKS; HOWLETT, 2013; PUGACH et al., 2016), uma vez que os dois receptores clássicos de estrogênio, ERa e $E R \beta$, são expressos no coração, tanto na fase adulta como na neonatal(GROHÉ et al., 1997).

A retirada dos ovários (ooforectomia) e a consequente diminuição dos níveis de estradiol podem levar a alterações importantes da função cardíaca, no que diz respeito à eletrofisiologia, contração e relaxamento do coração (PARKS; HOWLETT, 2013). Assim, estudos mostram que as propriedades elétricas do coração podem ser diretamente afetadas pela ausência do estrogênio (MCHUGH et al., 1995) contribuindo para a maior frequência de eventos arrítmicos no fim da vida reprodutiva. Neste sentido, um importante estudo mostrou que corações de cães induzidos à arritmia por isquemia e reperfusão, tiveram uma taxa significativamente menor de arritmias quando tratados previamente com estrogênio (MCHUGH et al., 1995). De modo 
semelhante, o 17-beta-estradiol marcou uma atividade antiarrítmica dosedependente, em corações de ratos, quando administrado 10 minutos antes da oclusão da artéria coronária (PHILP et al., 2006). Além disso, miócitos ventriculares, isolados de ratos, tratados com 17-beta-estradiol tiveram redução do pico de corrente de $\mathrm{Ca}^{+2}$ do tipo-L, de modo dependente de concentração (PHILP et al., 2006). Outros estudos mecanicistas ligaram a atividade antiarrítmica do estrogênio durante $\mathrm{I} / \mathrm{R}$ à abertura de canais de $\mathrm{K}^{+}$e ao aumento da liberação de óxido nítrico, que inibe o trocador $\mathrm{Na}^{+} / \mathrm{H}^{+}$no coração (OGITA et al., 2002; ANDERSON et al., 2005).

Alterações no estado contrátil do coração durante a sístole ou diástole também podem ser encontradas facilmente em ratas ooforectomizadas mostrando 0 impacto do estrogênio também sobre esses parâmetros cardíacos. Neste sentido, a diminuição do estrógeno em ratas ooforectomizadas promoveu uma menor resposta contrátil dos músculos papilares do ventrículo esquerdo, quando submetidos à estimulação adrenérgica, com menor expressão da proteína SERCA2 (cerca de 50\%) e aumento da fosfolamban (PLB) em relação aos animais controle; parâmetros estes que foram restaurados após a reposição com estrogênio (RIBEIRO et al., 2012).

Em relação à circulação coronariana, a queda na produção de hormônios ovarianos também está associada à maior incidência de doenças coronarianas, incluindo o infarto agudo do miocárdio (HU et al., 1999; HAK et al., 2000; GIUBERTI et al., 2007; ALMEIDA et al., 2014). Neste sentido, trabalhos experimentais demonstram que a ausência destes hormônios, após um evento de isquemia e reperfusão, está diretamente associado ao agravamento da disfunção autonômica, aumento do intervalo de tempo de contração e relaxamento ventricular, além de elevar a pressão diastólica final (GIUBERTI et al., 2007; LJ et al., 2010; XUE; XIAO; ZHANG, 2015). Apesar de tantas evidências quanto à importância dos hormônios ovarianos na função cardíaca, não podemos descartar o fato de, no final da vida reprodutiva, diminuições dos níveis dos hormônios ovarianos podem estar acompanhadas por outras condições, que por si só, podem também alterar a função cardíaca, como é o caso do hipotireoidismo subclínico (HAK et al., 2000; LEGRYS et al., 2013). Portanto, o desenvolvimento de trabalhos que avaliem os efeitos de cada uma dessas condições isoladamente, no intuito de descobrir qual o 
impacto de cada uma delas frente a um quadro de isquemia e reperfusão, podem contribuir com o avanço do conhecimento na área. 


\section{OBJETIVO}

O objetivo do presente estudo foi avaliar, em corações isolados de ratas Wistar adultas, os parâmetros de função cardíaca, após indução das seguintes condições experimentais.

-Diminuição dos níveis plasmáticos de hormônios tiroideanos, simulando o hipotireoidismo.

-Diminuição dos níveis plasmáticos de hormônios ovarianos, induzidos após ooforectomia. 


\section{CONCLUSÃO}

Com base nos resultados obtidos no presente estudo podemos concluir que:

- A ausência dos hormônios ovarianos por quatro semanas não altera a recuperação cardíaca após um evento de isquemia seguida de reperfusão.

- O hipotireoidismo subclínico não prejudica a recuperação funcional do coração após isquemia acompanhada de reperfusão.

- Os corações hipotireoideos podem ser mais resistentes à lesão por isquemia seguida de reperfusão, apesar de continuarem com a função cardíaca deprimida. 


\section{REFERÊNCIAS}

ABE, M.; OBATA, H.; TANAKA, H. Functional and metabolic responses to ischemia in the isolated perfused hypothyroid rat heart. Japanese circulation journal, v. 56, n. 7, p. 671-680, 1992.

ALMEIDA, S. A. De; ROBERTO, E.; CLAUDIO, G.; MENGAL, V. F.; GUEDES, $S$. Exercise Training Reduces Cardiac Dysfunction and Remodeling in Ovariectomized Rats Submitted to Myocardial Infarction. Plos One, v. 9, n. 12, p. 1-12, 2014.

ANDERSON, S. E.; KIRKLAND, D. M.; BEYSCHAU, A.; CALA, P. M.; STEVEN, E.; KIRKLAND, D. M.; BEYSCHAU, A. Acute effects of 17_-estradiol on myocardial $\mathrm{pH}, \mathrm{Na}$, and $\mathrm{Ca} 2$ and ischemia-reperfusion injury. American journal of physiology. Cell physiology, v. 8644, n. 9, p. 57-64, 2005.

ASARIAN, L.; GEARY, N. Sex differences in the physiology of eating. AJP: Regulatory, Integrative and Comparative Physiology, v. 305, n. 11, p. R1215-R1267, 2013.

* De acordo com:

ASSOCIAÇÃO BRASILEIRA DE NORMAS TÉCNICAS. NBR 6023: informação e documentação: Referencias: Elaboração. Rio de Janeiro, 2002. 
BERGER, L.; EL-ALFY, M.; LABRIE, F. Effects of intravaginal dehydroepiandrosterone on vaginal histomorphology, sex steroid receptor expression and cell proliferation in the rat.Journal of steroid biochemistry and molecular biology, v. 109, n. 1-2, p. 67-80, 2008.

BERS, D. M. Cardiac excitation-contraction coupling. Nature, v. 415, n. 6, p. 198-205, 2002.

BIONDI, B. MECHANISMS IN ENDOCRINOLOGY: Heart failure and thyroid dysfunction. European Journal of Endocrinology, v. 167, n. 5, p. 609-618, 2012.

BLAUSTEIN, J. D. An Estrogen by Any Other Name. Endocrinology, v. 149, n. 6, p. 2697-2698, jun. 2008.

BLENCK, C. L.; HARVEY, P. A.; RECKELHOFF, J. F.; LEINWAND, L. A. The Importance of Biological Sex and Estrogen in Rodent Models of Cardiovascular Health and Disease. Circulation Research, v. 118, n. 8, p. 1294-1312, 2016.

BULKLEY, G. B. Free radical-mediated reperfusion injury: a selective review. The British journal of cancer. Supplement, v. 8, p.2 66-73, 1987.

BUPHA-INTR, T.; WATTANAPERMPOOL, J.; PENA, J. R.; WOLSKA, B. M.; SOLARO, R. J. Myofilament response to $\mathrm{Ca} 2+$ and $\mathrm{Na}+\mathrm{H}+$ exchanger activity in sex hormone-related protection of cardiac myocytes from deactivation in hypercapnic acidosis. AJP: Regulatory, Integrative and Comparative Physiology, v. 292, n. 2, p. R837-R843, 28, 2006.

CANARIS, G. J.; MANOWITZ, N. R.; MAYOR, G.; RIDGWAY, E. C. The Colorado thyroid disease prevalence study. Archives of internal medicine, $v$. 160 , n. 4, p. 526-534, 2000.

COUSE, J. F.; KORACH, K. S.; KORACH, K. S. Estrogen Receptor Null Mice: What Have We Learned and Where Will They Lead Estrogen Receptor Null Mice: What Have We Learned and Where Will They Lead Us? Endocrine Reviews, v. 20, n. April, p. 358-417, 1999.

CROWLEY, W. F.; RIDGWAY, E. C.; BOUGH, E. W.; FRANCIS, G. S.; DANIELS, G. H.; KOURIDES, I. A.; MYERS, G. S.; MALOOF, F. Noninvasive evaluation of cardiac function in hypothyroidism. Response to gradual thyroxine replacement. The New England journal of medicine, v. 296, n. 1, p. 1-6, 1977.

CURCIO, C.; LOPES, A. M.; RIBEIRO, M. O.; FRANCOSO, O. A.; CARVALHO, S. D.; LIMA, F. B.; BICUDO, J. E.; BIANCO, A. C. Development of compensatory thermogenesis in response to overfeeding in hypothyroid rats. Endocrinology, v. 140, n. 8, p. 3438-3443, 1999.

DANTAS, A. P. V; FRANCO, M. D. C. P.; TOSTES, R. C. a; FORTES, Z. B.; COSTA, S. G.; NIGRO, D.; CARVALHO, M. H. C. Relative contribution of 
estrogen withdrawal and gonadotropins increase secondary to ovariectomy on prostaglandin generation in mesenteric microvessels. Journal of cardiovascular pharmacology, v. 43, n. 1, p. 48-55, 2004.

DIECKMAN, L. J.; SOLARO, R. J. Effect of thyroid status on thin-filament Ca2+ regulation and expression of troponin $I$ in perinatal and adult rat hearts. Circulation research, v. 67, n. 2, p. 344-351, 1990.

DILLMANN WH. Biochemical basis of thyroid hormone action in the heart. Am J Med. v.88. n. 88, p. 626-630, 1990.

DONG, J.; GAO, C.; LIU, J.; CAO, Y.; TIAN, L. TSH inhibits SERCA2a and the PKA / PLN pathway in rat cardiomyocytes. Oncotarget, v. 7, n. 26, 2016.

EBER, B. et al. Changes in thyroid hormone parameters after acute myocardial infarction. Cardiology (Switzerland), v. 86, n. 2, p. 152-156, 1995.

EMPSON, M. et al. Prevalence of thyroid disease in an older Australian population. Internal Medicine Journal, v. 37, n. 7, p. 448-455, 2007.

ESCOBAR-MORREALE, H. F.; OBREGON, M. J.; ESCOBAR DEL REY, F.; MORREALE DE ESCOBAR, G. Replacement therapy for hypothyroidism with thyroxine alone does not ensure euthyroidism in all tissues, as studied in thyroidectomized rats. Journal of Clinical Investigation, v. 96, n. 6, p. 28282838, 1995.

EVANS, H. M.; LONG, J. A. Characteristic Effects upon Growth, Oestrus and Ovulation Induced by the Intraperitoneal Administration of Fresh Anterior Hypophyseal Substance. Proceedings of the National Academy of Sciences of the United States of America, v. 8, n. 3, p. 38-39, 1922.

EVORA, P.R.B. ET AL. Lesão de Isquemia-Reperfusão. Aspectos Fisiopatológicos e a Importância da Função Endotelial. Arquivo Brasileiro Cardiologia, v.66, n.4, p. 239-245, 1996.

FRASIER, C. R.; BROWN, D. A.; SLOAN, R. C.; HAYES, B.; STEWART, L. M.; PATEL, H. D.; LUST, R. M.; ROSENBAUM, M. D. Stage of the estrous cycle does not influence myocardial ischemia-Reperfusion injury in rats (rattus norvegicus). Comparative Medicine, v. 63, n. 5, p. 416-421, 2013.

FRIBERG, L.; WERNER, S.; EGGERTSEN, G.; AHNVE, S. Rapid downregulation of thyroid hormones in acute myocardial infarction: is it cardioprotective in patients with angina? Archives of internal medicine, v. 162, n. 12, p. 1388-94, 2002.

FRIEHS, I.; EL NIDO, P. J. Increased susceptibility of hypertrophied hearts to ischemic injury. The Annals of Thoracic Surgery, v. 75, n. 2, p. S678-S684, 2003.

GIUBERTI, K.; PEREIRA, R. B.; BIANCHI, P. R.; PAIGEL, A. S.; VASSALLO, D. V.; STEFANON, I. Influence of Ovariectomy in the Right Ventricular Contractility in Heart Failure Rats. Archives of Medical Research, v. 38, n. 2, 
p. $170-175,2007$.

GOYAL, A.; SEMWAL, B. C.; YADAV, H. N. Abrogated cardioprotective effect of ischemic preconditioning in ovariectomized rat heart. Human \& experimental toxicology, v. 35, n. 6, p. 644-653, 2016.

GROHÉ, C.; KAHLERT, S.; LÖBBERT, K.; STIMPEL, M.; KARAS, R. H.; VETTER, H.; NEYSES, L. Cardiac myocytes and fibroblasts contain functional estrogen receptors.Federation of European Biochemical Societies, v. 416, n. 1, p. 107-112, 1997.

HAK, A. E.; POLS, H. A.; VISSER, T. J.; DREXHAGE, H. A.; HOFMAN, A.; WITTEMAN, J. C. Subclinical hypothyroidism is an independent risk factor for atherosclerosis and myocardial infarction in elderly women: the Rotterdam Study.Annales medicinae internae, v. 132, n. 4, p. 270-278, 2000.

HALL, J. M.; COUSE, J. F.; KORACH, K. S. The Multifaceted Mechanisms of Estradiol and Estrogen Receptor Signaling. Journal of Biological Chemistry, v. 276, n. 40, p. 36869-36872, 2001.

HARRIS, D. E.; WORK, S. S.; WRIGHT, R. K.; ALPERT, N. R.; WARSHAW, D. $M$. Smooth, cardiac and skeletal muscle myosin force and motion generation assessed by cross-bridge mechanical interactions in vitro. Journal of Muscle Research and Cell Motility, v. 15, n. 1, p. 11-19, 1994.

HOLLAND, F. W.; BROWN, P. S.; CLARK, R. E. Acute severe postischemic myocardial depression reversed by triiodothyronine. The Annals of Thoracic Surgery, v. 54, n. 2, p. 301-305, 1992.

HU, S.; REINCKE, M.; STRO, H.; HORN, M.; DIENESCH, C.; MORA, P.; SCHMIDT, H. H. H. W.; ALLOLIO, B.; NEUBAUER, S. Evidence Against a Role of Physiological Concentrations of Estrogen in Post-Myocardial Infarction Remodeling. Journal of the American College of Cardiology, v. 34, n. 5, p. 1427-1434, 1999.

IRIGOYEN, M. C.; PAULINI, J.; FLORES, L. J.; FLUES, K.; BERTAGNOLLI, M.; MOREIRA, E. D.; CONSOLIM-COLOMBO, F.; BELLO-KLEIN, A.; DE ANGELIS, K. Exercise training improves baroreflex sensitivity associated with oxidative stress reduction in ovariectomized rats. Hypertension, v. 46, n. 4, p. 998-1003, 2005.

KALOGERIS, T.; BAINES, C. P.; KRENZ, M.; KORTHUIS, R. J. Cell Biology of Ischemia/Reperfusion Injury.International review of cell and molecular biology, v. 298, n. 3, p. 229-317, 2012.

KANNEL, W. B.; HJORTLAND, M. C.; MCNAMARA, P. M.; GORDON, T. Menopause and risk of cardiovascular disease: the Framingham study. Annals of internal medicine, v. 85, n. 4, p. 447-452, 1976.

KARTHICK, N.; DILLARA, K.; POORNIMA, K. N.; SUBHASINI, A. S. Dyslipidaemic changes in women with subclinical hypothyroidism. Journal of Clinical and Diagnostic Research, v. 7, n. 10, p. 2122-2125, 2013. 
KENESSEY, A.; OJAMAA, K. Thyroid hormone stimulates protein synthesis in the cardiomyocyte by activating the Akt-mTOR and p70S6K pathways. Journal of Biological Chemistry, v. 281, n. 30, p. 20666-20672, 2006.

KIM, Y.; CHEN, B.; BEAUREGARD; KOURETAS, P.; THOMAS, G.; FARHAT, M.; MYERS, A.; DE, L. 17 $\beta$-Estradiol Prevents Dysfunction of Canine Coronary Endothelium. Circulation, v. 94, n. 12, p. 2901-2908, 1996.

KLEIN, I.; DANZI, S. Thyroid disease and the heart Circulation. Circulation, v. 116, n. 15, p. 1725-1735, 2007.

KLEIN, I. Hipertro a cardíaca induzida por tiroxina: tempo de desenvolvimento e inibição por Propranolol * Oxford Academic account. Endocrinology, v. 123, n. 1, p. 203-210, 1988.

KLEIN, L. E.; SIGEL, a V; DOUGLAS, J. a; EGHBALI-WEBB, M. Upregulation of collagen type I gene expression in the ventricular myocardium of thyroidectomized male and female rats. Journal of molecular and cellular cardiology, v. 28, n. 1, p. 33-42, 1996

KNOWLTON, A. A.; KORZICK, D. H. Estrogen and the female heart. Molecular and Cellular Endocrinology, v. 389, n. 1, p. 31-39, 2014.

KONG, L.; WEI, Q.; FEDAIL, J. S.; SHI, F.; NAGAOKA, K.; WATANABE, G. Effects of thyroid hormones on the antioxidative status in the uterus of young adult rats. Journal of Reproduction and Development, v. 61, n. 3, p. 219227, 2015.

KORZICK, D. H.; LANCASTER, T. S. Age-related differences in cardiac ischemia-reperfusion injury: effects of estrogen deficiency. Pflügers Archiv European Journal of Physiology, v. 465, n. 5, p. 669-685, 2013.

KRENZ, M.; ROBBINS, J. Impact of beta-myosin heavy chain expression on cardiac function during stress. Journal of the American College of Cardiology, v. 44, n. 12, p. 2390-2397, 2004.

LEDEE, D.; PORTMAN, M. A.; KAJIMOTO, M.; ISERN, N.; OLSON, A. K. Thyroid Hormone Reverses Aging-Induced Myocardial Fatty Acid Oxidation Defects and Improves the Response to Acutely Increased Afterload. PLoS ONE, v. 8, n. 6, 2013.

LEGRYS, V. a; FUNK, M. J.; LORENZ, C. E.; GIRI, A.; JACKSON, R. D.; MANSON, J. E.; SCHECTMAN, R.; EDWARDS, T. L.; HEISS, G.; HARTMANN, K. E. Subclinical hypothyroidism and risk for incident myocardial infarction among postmenopausal women. The Journal of clinical endocrinology and metabolism, v. 98, n. 6, p. 2308-2317, 2013.

LI, X.; HUANG, J.; YI, P.; BAMBARA, R. a; HILF, R.; MUYAN, M. Single-Chain Estrogen Receptors ( ERs ) Reveal that the ER a / $\beta$ Heterodimer Emulates Functions of the ER a Dimer in Genomic Estrogen Signaling Pathways Single- 
Chain Estrogen Receptors ( ERs ) Reveal that the ER, Heterodimer Emulates Functions of the. Molecular and cellular biology, v. 24, n. 17, p. 7681-7694, 2004.

LIU, D.; TENG, W.; SHAN, Z.; YU, X.; GAO, Y.; WANG, S.; FAN, C.; WANG, H.; ZHANG, $H$. The effect of maternal subclinical hypothyroidism during pregnancy on brain development in rat offspring. Thyroid, v. 20, n. 8, p. 909915, 2010.

LJ, F.; FIGUEROA, D.; IC, S.; JORGE, L.; MC, I.; RODRIGUES, B.; K, D. A. Effects of exercise training on autonomic dysfunction management in an experimental model of menopause and myocardial infarction . Menopause, $v$. 17, n. 4, p. 712-717, 2010.

LONDOÑO, Á. L.; GALLEGO, M. L.; BAYONA, A.; LANDÁZURI, P. Prevalencia de hipotiroidismo y relación con niveles elevados de anticuerpos antiperoxidasa y yoduria en población de 35 y más años en Armenia. 2009-2010. Revista de Salud Pública, v. 13, n. 6, p. 998-1009, 2011.

LU, L.; YU, X.; TENG, W.; SHAN, Z. Treatment with levothyroxine in pregnant rats with subclinical hypothyroidism improves cell migration in the developing brain of the progeny. Journal of Endocrinological Investigation, v. 35, n. 5, p. 490-496, 2012.

MADATHIL, A.; HOLLINGSWORTH, K. G.; BLAMIRE, A. M.; RAZVI, S.; NEWTON, J. L.; TAYLOR, R.; WEAVER, J. U. Levothyroxine improves abnormal cardiac bioenergetics in subclinical hypothyroidism: A cardiac magnetic resonance spectroscopic study. Journal of Clinical Endocrinology and Metabolism, v. 100, n. 4, p. E607-E610, 2015.

MANDL, A. M. The Phases of the Oestrous Cycle in the Adult White Rat. Journal of Experimental Biology, v. 28, n. 4, p. 576-584, 1951.

MANO, T.; SAKAMOTO, H.; FUJITA, K.; MAKINO, M.; KAKIZAWA, H.; NAGATA, M.; KOTAKE, M.; HAMADA, M.; UCHIMURA, K.; HAYAKAWA, N.; HAYASHI, R.; NAKAI, A.; ITOH, M.; KUZUYA, H.; NAGASAKA, A. Effects of thyroid hormone on catecholamine and its metabolite concentrations in rat cardiac muscle and cerebral cortex. Thyroid, v. 8, n. 4, p. 353-358, 1998.

MARCONDES, F. K.; BIANCHI, F. .; TANNO, A. . Determination of The Estrous Cycle Phases of Rats: Some Helpful Considerations. Brazilian Journal Biology, v. 62, n. 4, p. 609-614, 2002.

MAZER, N. A. Interaction of Estrogen Therapy and Thyroid Hormone Replacement in Postmenopausal Women. Thyroid, v. 14, p.27-34, 2004.

MCHUGH, N. A.; COOK, S. M.; SCHAIRER, J. L.; BIDGOLI, M. M.; MERRILL, G. F. Ischemia- and reperfusion-induced ventricular arrhythmias in dogs: effects of estrogen. The American journal of physiology, v. 268, n. 2, p. H2569H2573, 1995.

MCNULTY, P. H.; JAGASIA, D.; WHITING, J. M.; CAULIN-GLASER, T. Effect 
of 6-wk estrogen withdrawal or replacement on myocardial ischemic tolerance in rats. American journal of physiology. Heart and circulatory physiology, v. 278, n. 4, p. H1030- $\mathrm{H} 1034,2000$.

MEENA, C. L.; MEENA, R. D.; NAWAL, R.; MEENA, V. K.; BHARTI, A.; MEENA, L. P. Assessment of left ventricular diastolic dysfunction in sub-clinical hypothyroidism. Acta Informatica Medica, v. 20, n. 4, p. 218-220, 2012.

MELI, R.; PACILIO, M.; RASO, G. M.; ESPOSITO, E.; COPPOLA, A.; NASTI, A.; DI CARLO, C.; NAPPI, C.; DI CARLO, R. Estrogen and raloxifene modulate leptin and its receptor in hypothalamus and adipose tissue from ovariectomized rats. Endocrinology, v. 145, n. 7, p. 3115-3121, 2004.

MITTAL, G.; CHANDRAIAH, G.; RAMARAO, P.; RAVI KUMAR, M. N. V. Pharmacodynamic evaluation of oral estradiol nanoparticles in estrogen deficient (ovariectomized) high-fat diet induced hyperlipidemic rat model. Pharmaceutical Research, v. 26, n. 1, p. 218-223, 2009.

MONZANI, F.; DI BELLO, V.; CARACCIO, N.; BERTINI, A.; GIORGI, D.; GIUSTI, C.; FERRANNINI, E. Effect of levothyroxine on cardiac function and structure in subclinical hypothyroidism: A double blind, placebo-controlled study. Journal of Clinical Endocrinology and Metabolism, v. 86, n. 3, p. 11101115, 2001.

MOUROUZIS, I.; DIMOPOULOS, A.; SARANTEAS, T.; TSINARAKIS, N.; LIVADAROU, E.; SPANOU, D.; KOKKINOS, A. D.; XINARIS, C.; PANTOS, C.; COKKINOS, D. V. Ischemic preconditioning fails to confer additional protection against ischemia-reperfusion injury in the hypothyroid rat heart. Physiological Research, v. 58, n. 1, p. 29-38, 2009.

NAKAMURA, H.; PJ, del N.; E, J.; SARIN, M.; H, F.; S, L. Age related differences in cardiac susceptibility to ischemia / reperfusion injury. Response to deferoxamine. Journal of Thoracic and Cardiovascular Surgery, v. 104, n. 1 , p. $165-172,1992$.

NILSSON, S.; GUSTAFSSON, J.-Å. Estrogen Receptors: Therapies Targeted to Receptor Subtypes. Clinical Pharmacology \& Therapeutics, v. 89, n. 1, p. 44-55, 2011.

OGITA, H.; NODE, K.; ASANUMA, H.; SANADA, S.; LIAO, Y. Amelioration of Ischemia- and Reperfusion-Induced Myocardial Injury by the Selective Estrogen Receptor Modulator, Raloxifene, in the Canine Heart. Journal of the American College of Cardiology, v. 40, n. 5, p. 2-9, 2002.

OHYAMA, T.; NAKAI, A.; NAGASAKA, A.; AONO, T.; MASUNAGA, R.; NAKAGAWA, H.; KATAOKA, K.; OHTANI, S.; SHINODA, S.; IWASE, K. [Change in serum thyroid hormone levels in patients with acute myocardial infarction]. Nihon Naibunpi Gakkai zasshi, v. 63, n. 1, p. 19-25, 1987.

OJAMAA, K.; KENESSEY, A; KLEIN, I. Thyroid hormone regulation of phospholamban phosphorylation in the rat heart. Endocrinology, v. 141, n. 6, p. 2139-2144, 2000. 
PANTOS C, VASSILIKI V, DENNIS D C, DENNIS V. Thyroid hormone and phenotypes of cardioprotection. Basic Res Cardiol. v.99, n. 3, p. 101-120, 2004.

PANTOS, C.; MALLIOPOULOU, V.; MOUROUZIS, I.; SFAKIANOUDIS, K.; TZEIS, S.; DOUMBA, P.; XINARIS, C.; COKKINOS, A. D.; CARAGEORGIOU, H.; VARONOS, D. D.; COKKINOS, D. V. Propylthiouracil-induce hypothyroidism is associated with increased tolerance of the isolated rat heart to ischaemiareperfusion. Journal of Endocrinology, v. 178, n. 3, p. 427-435, 2003.

PANTOS, C.; MOUROUZIS, I.; MALLIOPOULOU, V.; PAIZIS, I.; TZEIS, S.; MORAITIS, P.; SFAKIANOUDIS, K.; VARONOS, D. D.; COKKINOS, D. V. Dronedarone Administration Prevents Body Weight Gain and Increases Tolerance of the Heart to Ischemic Stress: A Possible Involvement of Thyroid Hormone Receptor a 1. Thyroid, v. 15, n. 1, p. 16-23, jan. 2005.

PARKS, R. J.; HOWLETT, S. E. Sex differences in mechanisms of cardiac excitation-contraction coupling.Pflügers Archiv - European Journal of Physiology, v. 465, n. 5, p. 747-763, 2013.

PHILP, K. L.; HUSSAIN, M.; BYRNE, N. F.; DIVER, M. J.; HART, G.; COKER, S. J. Greater antiarrhythmic activity of acute $17 \mathrm{~b}$-estradiol in female than male anaesthetized rats : correlation with $\mathrm{Ca} 2 \mathrm{p}$ channel blockade.British Journal of Pharmacology, v. 149, n. 10, p. 233-242, 2006.

PUGACH, E. K.; BLENCK, C. L.; DRAGAVON, J. M.; LANGER, S. J.; LEINWAND, L. A. Estrogen receptor profiling and activity in cardiac myocytes. Molecular and Cellular Endocrinology, v. 431, p. 62-70, 2016.

REVANKAR, C. M. A Transmembrane Intracellular Estrogen Receptor Mediates Rapid Cell Signaling. Science, v. 307, n. 5715, p. 1625-1630, 11 mar. 2005.

RIBEIRO, jr R. F.; BM, P.; FF, P.; FIORIM, J.; FM, D.; FL, L.; AA, F.; DV, V. Myocardial contractile dysfunction induced by ovariectomy requires AT1 receptor activation in female rats. Cell Physiol Biochem, v. 30, n. 1, p. 1-12, 2012.

ROGER, V. L.; GO, A. S.; LLOYD-JONES, D. M., et al. Heart Disease and Stroke Statistics-2011 Update: A Report From the American Heart Association. Circulation, v. 123, n. 4, p. e18-e209, 12011.

SANDLER, B.; WEBB, P.; APRILETTI, J. W.; HUBER, B. R.; TOGASHI, M.; CUNHA LIMA, S. T.; JURIC, S.; NILSSON, S.; WAGNER, R.; FLETTERICK, R. J.; BAXTER, J. D. Thyroxine-thyroid hormone receptor interactions. The Journal of Biological Chemistry, v. 279, n. 53, p. 55801-55808, 2004.

SAPIN, R.; SCHLIENGER, J. L. Dosages de thyroxine (T4) et tri-iodothyronine (T3): techniques et place dans le bilan thyroïdien fonctionnel. Annales de Biologie Clinique, v 61, n. 4, p. 411- 420, 2003.

SCHMIDT-OTT, U. M.; ASCHEIM, D. D. Thyroid Hormone and Heart Failure. Current Heart Failure Reports, v. 3, n. 3, p. 114-119, 2006. 
SHAHRIVAR, F. F.; BADAVI, M.; DIANAT, M.; MARD, A.; AHANGARPOUR, A.; SAMARBAF-ZADEH, A. Exogenous apelin changes alpha and beta myosin heavy chain mRNA expression and improves cardiac function in PTU-induced hypothyroid rats. Gene, v. 595, p. 25-30, 2016.

SHAIKH, A. a. Estrone and Estradiol Levels in the Ovarian Venous Blood from Rats During the Estrous Cycle and Pregnancy. Biology of Reproduction, v. 307, p. 297-307, 1971.

SHIBLI-RAHHAL, A.; HAYNES, W.; SINKEY, C.; DILLON, J. S. Effect of dehydroepiandrosterone ( DHEA ) on vascular function in postmenopausal women with diabetes: a ran- domized controlled trial. Journal of Diabetes Research and Clinical Metabolism, v. 1, n. 1, p. 13, 2012.

SUGIURA, S.; KOBAYAKAWA, N.; FUJITA, H.; YAMASHITA, H.; MOMOMURA, S.; CHAEN, S.; OMATA, M.; SUGI, H. Comparison of unitary displacements and forces between 2 cardiac myosin isoforms by the optical trap technique: molecular basis for cardiac adaptation. Circulation Research, v. 82, n. 10, p. 1029-1034, 1998.

SUZUKI, A.; ENARI, M.; ABE, Y.; OHTA, Y.; IGUCHI, T. Effect of ovariectomy on histological change and protein expression in female mouse reproductive tracts. In Vivo, v. 10, n. 1, p. 103-110, 1996a.

SUZUKI, A.; ENARI, M.; HAYASHI, Y.; OHTA, Y.; KOSHIKAWA, N.; MIYAZAKI, K.; IGUCHI, T. Characterization and Role of Proteinases Induced by EstrogenDeprivation in Female Mouse Reproductive Tracts. Reproductive Toxicology, v. 10 , n. 2, p. 129-135, 1996b.

TAVARES, F. M.; DA SILVA, I. B.; GOMES, D. A.; BARRETO-CHAVES, M. L. $M$. Angiotensin II type 2 receptor (AT2R) is associated with increased tolerance of the hyperthyroid heart to ischemia-reperfusion. Cardiovascular Drugs and Therapy, v. 27, n. 5, p. 393-402, 2013.

THOMAS, S. A.; PALMITER, R. D. Thermoregulatory and metabolic phenotypes of mice lacking noradrenaline and adrenaline. nature, v. 387, n. 6628, p. 94-97, 1 maio 1997.

VAN DER VELDEN, J. Diastolic myofilament dysfunction in the failing human heart. Pflugers Archiv European Journal of Physiology,v. 462, n. 1, p. 155163, 2013.

WILLEMS, L.; ZATTA, A.; HOLMGREN, K.; ASHTON, K. J.; HEADRICK, J. P. Age-related changes in ischemic tolerance in male and female mouse hearts. Journal of Molecular and Cellular Cardiology, v. 38, n. 2, p. 245-256, 2005.

WONG, C. M.; YAO, X.; AU, C. L.; TSANG, S. Y.; FUNG, K. P.; LAHER, I.; VANHOUTTE, P. M.; HUANG, Y. Raloxifene prevents endothelial dysfunction in aging ovariectomized female rats. Vascular Pharmacology, v. 44, n. 5, p. 290298, 2006.

XUE, Q.; XIAO, D.; ZHANG, L. Estrogen Regulates Angiotensin II Receptor 
Expression Patterns and Protects the Heart from Ischemic Injury in Female Rats.Biology of Reproduction, v. 93, n. May, p. 1-9, 2015.

YANG, S. S.; TANG, L.; LI, R. G.; GE, G. H.; QU, X. K.; MA, J. W.; QIAO, Z. Y.; ZHANG, L.; LIU, H. J.; HOU, Y. M.; CAO, H.; HAO, Z. M.; CHENG, W. B.; WANG, H. W. The effects of subdinical hypothyroidism on serum lipid level and TLR4 expression of monocyte in peripheral blood of rats. Neuroendocrinology Letters, v. 35, n. 1, p. 80-86, 2014.

YANG, S.; CHOUDHRY, M. A.; HSIEH, Y.-C.; HU, S.; RUE, L. W.; BLAND, K. I.; CHAUDRY, I. H. Estrus cycle: influence on cardiac function following traumahemorrhage. American journal of physiology. Heart and circulatory physiology, v. 291, n. 6, p. H2807- H2815, 2006.

YOUSEFZADEH, N.; JEDDI, S.; ALIPOUR, M. R. Effect of Fetal Hypothyroidism on Cardiac Myosin Heavy Chain Expression in Male Rats. Arquivos Brasileiros de Cardiologia, v. 107, n. 2, 2016.

ZHANG, L.; PARRATT, J. R.; BEASTALL, G. H.; PYNE, N. J.; FURMAN, B. L. Streptozotocin diabetes protects against arrhythmias in rat isolated hearts: Role of hypothyroidism. European Journal of Pharmacology, v. 435, n. 2, p. 269276, 2002. 